\title{
HIGHLIGHTS
}

BLADDER CANCER

\section{Optical coherence tomography improves UCC detection}

Targeted optical coherence tomography (OCT) in combination with hexaminolevulinate (HAL) fluorescence cystoscopy improves diagnostic accuracy in detecting urothelial cell carcinoma (UCC) of the bladder, report Schmidbauer and colleagues from the Medical

University of Vienna.

Fluorescence cystoscopy has been shown to significantly improve detection of UCC when used as an adjunct to white-light cystoscopy. HAL fluorescence cystoscopy shows higher sensitivity than white-light cystoscopy in detecting bladder cancer, but has a much higher false-positive rate. OCT is a noninvasive, high-resolution, cross-sectional tissue imaging method that measures optical scattering of light.

The study aimed to evaluate whether adding targeted OCT analysis of lesions identified as suspicious by whitelight cystoscopy and HAL fluorescence cystoscopy would improve diagnostic accuracy in detecting UCC. The study group incorporated 66 patients with suspected bladder cancer. A total of 232 lesions previously identified by white-light and HAL cystoscopy were analyzed by OCT and subsequently resected or biopsied.

UCC was diagnosed in 58 patients $(88 \%)$. Per-patient sensitivity with whitelight cystoscopy was $89.7 \%$, and $100 \%$ for both HAL fluorescence cystoscopy alone and HAL cystoscopy with OCT. Per-lesion sensitivity and specificity were $69.3 \%$ and $83.7 \%$, respectively, for white-light, $97.5 \%$ and $78.6 \%$ for HAL cystoscopy alone, and $97.5 \%$ and $97.9 \%$ for HAL cystoscopy with OCT. The combined false-positive rate for white-light and HAL cystoscopy was $23 \%$, while the false-positive rate with HAL cystoscopy alone was $36 \%$ for suspicious flat lesions. OCT confirmed nonmalignant histology in 36 of 42 benign fluorescent flat lesions.
The researchers conclude that using HAL fluorescence cystoscopy in combination with OCT increases the specificity of fluorescence cystoscopy in targeting UCC by $19 \%$ at the lesion level, and by $25 \%$ per patient. They also state that this combination enables prediction of invasion, and allows more-precise identification of suspicious flat lesions. This approach enables the user to identify suspicious areas for closer inspection by OCT, thereby reducing the incidence of unnecessary biopsies. OCT is limited, however, by poor visualization of flat lesions in white-light cystoscopy; therefore, time-consuming multiple measurements are required.

\section{Rebecca Drake}

Original article Schmidbauer, J. et al. Fluorescence cystoscopy with high-resolution optical coherence tomography imaging as an adjunct reduces false-positive findings in the diagnosis of urothelial carcinoma of the bladder. Eur. Urol. 56, 914-919 (2009) 